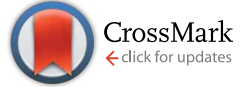

Cite this: J. Mater. Chem. A, 2014, 2, 12703

Received 5th May 2014 Accepted 18th June 2014

DOI: $10.1039 / c 4 t a 02260 b$

\section{Direct synthesis of carbide-derived carbon monoliths with hierarchical pore design by hard- templating $\dagger$}

\author{
Winfried Nickel, ${ }^{a}$ Martin Oschatz, ${ }^{a}$ Martin von der Lehr, ${ }^{\mathrm{b}}$ Matthias Leistner, ${ }^{\mathrm{c}}$ \\ Guang-Ping Hao, ${ }^{a}$ Philipp Adelhelm, ${ }^{b}$ Philipp Müller, ${ }^{a}$ Bernd M. Smarsly ${ }^{b}$ \\ and Stefan Kaskel ${ }^{\star a c}$
}

www.rsc.org/MaterialsA

\begin{abstract}
Carbide-derived carbon Monoliths (CDC-Ms) containing a multimodal arrangement with high volumes of micro- meso- and macropores are prepared by direct nanocasting of silica monoliths with polycarbosilane precursors. CDC-Ms show well-defined pore structures along with specific surface areas of more than $2600 \mathrm{~m}^{2} \mathrm{~g}^{-1}$ and overall pore volumes as high as $3.14 \mathrm{~cm}^{3} \mathrm{~g}^{-1}$. They exhibit advanced gas filtration properties compared to purely microporous materials due to enhanced storage capacities and kinetics as demonstrated by thermal response measurements based on InfraSORP technology.
\end{abstract}

Carbide-derived carbons (CDCs) are a novel class of nanoporous carbon materials produced by the selective removal of metal- or semi-metal atoms from carbides by high-temperature halogenation or thermal/hydrothermal decomposition. ${ }^{1}$ CDCs have received considerable attention due to their large number of potential applications such as gas storage, ${ }^{2}$ gas separation,,$^{3,4}$ catalysis, ${ }^{5}$ removal of cytokines from blood plasma, ${ }^{6}$ and as electrode material in electrochemical double layer capacitors (EDLCs) ${ }^{7-9}$ or rechargeable batteries. ${ }^{\mathbf{1 0 , 1 1}}$ Their specific advantage over other microporous carbon materials (i.e. activated carbons) is the precisely controllable pore diameter by using different carbide precursors and synthesis temperatures. ${ }^{2}$ For that reason, they are intensively applied as model substances for investigations on ion- and gas adsorption phenomena in porous solids. ${ }^{2,12-15}$ The low oxygen content of these carbon materials is related to their production in oxygen-free atmosphere allowing to further tune their surface properties for a

\footnotetext{
${ }^{a}$ Department of Inorganic Chemistry, Dresden University of Technology, Bergstraße 66, D-01062 Dresden, Germany. E-mail: stefan.kaskel@chemie.tu-dresden.de

${ }^{b}$ Institute of Physical Chemistry, Justus-Liebig-University Gießen, Heinrich-Buff-Ring 58, D-35392 Gießen, Germany

${ }^{c}$ Fraunhofer Institut für Werkstoff und Strahltechnik (IWS), Winterbergstraße 28, D01277 Dresden, Germany

$\dagger$ Electronic supplementary Information (ESI) available: Synthesis scheme and TGA curve of CDC-Ms, QSDFT pore size analysis, SEM images of the template, characterization of the reference material, further thermal response measurement data, and experimental details. See DOI: 10.1039/c4ta02260b
}

given application. ${ }^{\mathbf{1 6}}$ Depending on morphology of the carbide precursors, CDCs can be prepared in different textures and morphologies such as powders, ${ }^{2}$ nanopowders, ${ }^{17}$ thin films, ${ }^{18}$ fibers $^{19}$ and biomorphic ceramics. ${ }^{20}$ Those carbons can reach very high specific surface areas above $2000 \mathrm{~m}^{2} \mathrm{~g}^{-1}$ and the porosity can be further enhanced by post-synthetic activation procedures. $^{21}$ However, the strictly microporous character of CDCs is a drawback in applications requiring the presence of an interconnected hierarchical pore structure to ensure both high capacities (provided by micro- and narrow mesopores) as well as efficient transport throughout the entire material (provided by larger transport pores). Besides the use of precursors with a low carbon content/free metal phase ${ }^{22,23}$ and the use of polyureasilazane precursors, ${ }^{24}$ suitable templating strategies display noteworthy alternatives for the synthesis of carbide-derived carbons with hierarchical pore designs. The evaporation induced self-assembly (EISA) approach $^{25}$ can be used for the synthesis of free-standing films of ordered mesoporous CDCs (OM-CDCs) starting from resin and metal oxide precursors followed by carbothermal reduction and high-temperature chlorine treatment. ${ }^{26}$ Alternatively, hard-templating (also referred to as "nanocasting") of mesoporous silica templates with polycarbosilane (PCS) precursors can be applied to synthesize hierarchically structured CDC powders containing micro- and mesopores along with outstanding specific surface areas of up to $2900 \mathrm{~m}^{2} \mathrm{~g}^{-1}$ and total pore volumes as high as $2.6 \mathrm{~cm}^{3} \mathrm{~g}^{-1} \cdot{ }^{11}$

Recently, there has been much effort to synthesize CDC materials with monolithic appearance (CDC-Ms) due to the importance of shaping for practical applications as well as the increased volumetric efficiency of monoliths compared to powders. For instance, Yeon and co-workers reported the synthesis of monolithic TiC-derived carbons with a $58 \%$ and $50 \%$ higher storage capacity for methane and hydrogen, respectively, ${ }^{27}$ when compared to $\mathrm{CDC}$ powder with similar specific surface area and average pore size. CDC-Ms featuring a hierarchical pore system can be obtained by binder-assisted compression of ordered mesoporous silica/PCS composites followed by pyrolysis, template removal in hydrofluoric acid 
solution and high-temperature chlorine treatment yielding CDC-Ms containing a bimodal micro-mesopore system. ${ }^{28}$ The fabrication of CDC-Ms with multimodal pore architecture of micro- meso- and macropores can be achieved by a soft-templating approach starting from polymerized high internal phase emulsions (PolyHIPEs). ${ }^{3}$ The resulting CDCs are characterized by high specific surface areas of more than $2300 \mathrm{~m}^{2} \mathrm{~g}^{-1}$ coupled with overall pore volumes as high as $8.5 \mathrm{~cm}^{3} \mathrm{~g}^{-1}$. The efficient gas filtration properties of these materials are due to the simultaneous presence of large amounts of micro- and mesopores and a distinct transport system of larger voids. Despite the advantages of the highly open pore structure and the binderfree synthesis, these CDC-Ms do not contain well-defined macropores, and therefore mass transport might be of different magnitude at different positions within the monolith. In addition, these materials lack a large amount of interconnected mesopores, which are of particular importance for the effective adsorption of large molecules, such as enzymes or dyes.

Herein, the synthesis of CDC-Ms is presented using nanocasting of hierarchically meso-macroporous silica monoliths starting from tetramethoxysilane as $\mathrm{SiO}_{2}$ precursor. The $\mathrm{SiO}_{2}$ monoliths show a distinct 3D connected structure of approximately 1-2 $\mu \mathrm{m}$ macropores containing mesopores of 5-10 $\mathrm{nm}$ in diameter within their walls and are tailored to achieve high homogeneity and excellent mass transport characteristics for liquid phase separation applications (chromatography). ${ }^{29}$ Such monoliths were transformed into corresponding meso- and macroporous carbons by replication with precursors such as mesophase pitch. ${ }^{30,31}$ However, for gas filtration applications a high degree of microporosity in parallel to mass transport optimized meso- and macroporosity is desired. In order to achieve that goal we have used the polymer-precursor CDC approach described above. In the first step, the monoliths are infiltrated with liquid allylhydrido-polycarbosilane (SMP-10, Starfire Systems). After vacuum-assisted infiltration of PCS the resulting composites are transferred to silicon carbide replicas of the template by pyrolysis under argon atmosphere at a maximum temperature of $800^{\circ} \mathrm{C}$. Removal of the silica template is followed by the insertion of a large volume of micropores by selective removal of the silicon atoms in an atmosphere of hot chlorine gas (ESI, Fig. S1†). The monolithic appearance of the silica templates can be kept intact over the entire synthesis pathway (Fig. S1†). A linear shrinkage of $\sim 20 \%$ is observed during the conversion from polymer to the silicon carbide while the high-temperature chlorination step is fully conformal, i.e. the texture of the silicon carbide is precisely transformed into CDC-Ms. Quantitative silicon removal is detected by electron dispersive X-ray spectroscopy (EDX) measurements. The carbon content in the CDCs is 99.9 atom\% while any potential impurities of silicon, oxygen or chlorine are below the detection limit. Accordingly, thermogravimetric analysis of the carbon materials under oxidative conditions (air atmosphere) leads to quantitative carbon combustion proving the complete removal of silicon during chlorine treatment (Fig. S2 $\dagger$ ).

While the pristine silica templates show a distinct mesoporosity with high specific surface area of $680 \mathrm{~m}^{2} \mathrm{~g}^{-1}$ and a mesopore volume of $1.01 \mathrm{~cm}^{3} \mathrm{~g}^{-1}$ before PCS infiltration (Fig. 1 and Table S1 $\dagger$ ), after pyrolysis the porosity values of the infiltrated monoliths are close to zero because of the complete filling of the pore systems (Fig. 1). Removal of the template in hydrofluoric acid solution leads to the formation of a mesoporous silicon carbide material with a specific surface area of $365 \mathrm{~m}^{2} \mathrm{~g}^{-1}$ and a total pore volume of $0.38 \mathrm{~cm}^{3} \mathrm{~g}^{-1}$ (Fig. 1 and Table $\mathrm{S} 1 \dagger)$. Etching of the silicon atoms by high-temperature chlorine treatment leads to the implementation of a large volume of micropores (cumulative QSDFT micropore volume $=0.6 \mathrm{~cm}^{3} \mathrm{~g}^{-1}$ ) and the specific surface area significantly increases to $2662 \mathrm{~m}^{2} \mathrm{~g}^{-1}$ (Table $\mathrm{S} 1 \dagger$ ). Since the carbide-tocarbon transformation is highly conformal, the mesopore system provided by the silicon carbide precursors is fully recovered as indicated by the narrow hysteresis loop in the nitrogen physisorption isotherm of the CDC monoliths associated with a high uptake of nitrogen at $P / P_{0}=0.4-0.8$ leading to a high total micro-mesopore volume of $2.28 \mathrm{~cm}^{3} \mathrm{~g}^{-1}$ (calculated at $P / P_{0}=0.99$ ).

In accordance to the shape of the nitrogen physisorption isotherms, the quenched solid density functional theory (QSDFT) pore size analysis of a low-pressure measurement (Fig. S3†) shows the presence of micropores of $1.0 \mathrm{~nm}$ in size as it is typical for polycarbosilane-derived CDC-Ms prepared at comparable temperature. The mesopores in the CDC monoliths are narrowly distributed in the range of 3-12 $\mathrm{nm}$ and centred at a diameter of $6.5 \mathrm{~nm}$. The good agreement between the used DFT kernel (nitrogen on carbon containing slit/cylindrical pores, adsorption branch) and the experimental data shows that this QSDFT method calculates a reliable pore size distribution (PSD; Fig. S4 $\dagger$ ). We note that the PSDs from the adsorption and desorption branches of the isotherm do not significantly differ in size and volume of the pores, i.e. desorption of nitrogen from the mesopores takes place under equilibrium conditions. While the presence of minor cavitation effects cannot be ruled out due to the slight step in the desorption branch at $P / P_{0}=0.42$, the huge majority of the mesopores in the CDC monoliths empty under equilibrium conditions indicating their advanced

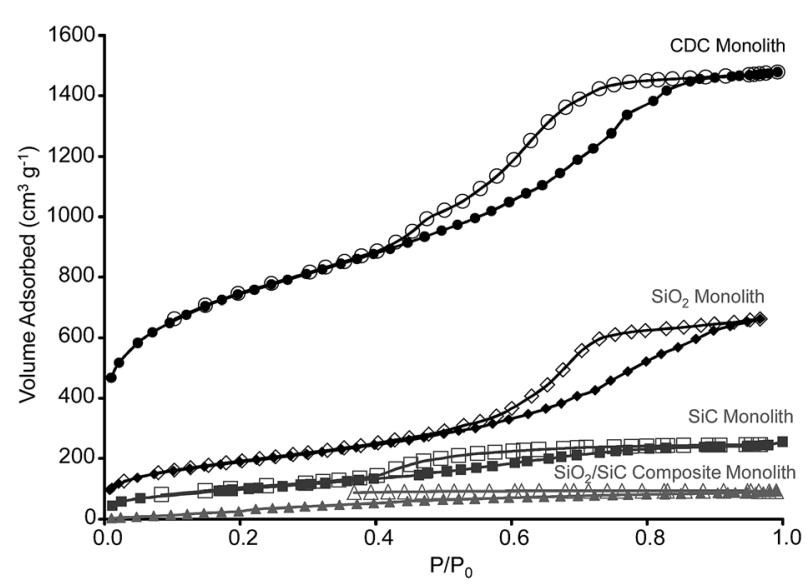

Fig. 1 Nitrogen adsorption/desorption (filled symbols/empty symbols) isotherms of the $\mathrm{CDC}$ monoliths (circles), $\mathrm{SiO}_{2}$ monoliths (diamonds), $\mathrm{SiC}$ monoliths (squares), and $\mathrm{SiO}_{2} / \mathrm{SiC}$ composite monoliths (triangles) measured at $-196^{\circ} \mathrm{C}$. 


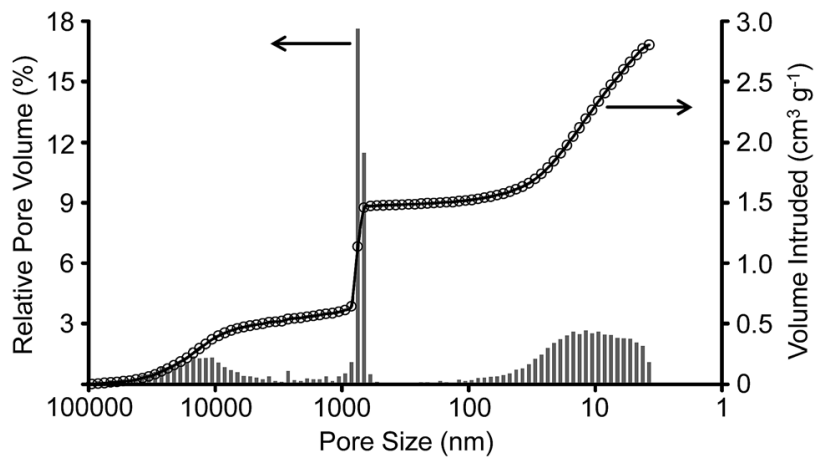

Fig. 2 Mercury intrusion curves (black circles) and relative pore volumes of the increments (grey stacks) of the CDC monoliths.

accessibility in contrast to previously reported hierarchical CDC materials with comparable pore size obtained by nanocasting. ${ }^{11}$

The larger pores in the CDC monoliths were characterized by mercury intrusion porosimetry (Fig. 2). While the intrusion $\left(0.5 \mathrm{~cm}^{3} \mathrm{~g}^{-1}\right)$ at low pressures (i.e. at larger pore size) might be related to inter-particular spaces, the sharp increase of the intruded volume at a pore size of $750 \mathrm{~nm}$ shows that the macropores in the CDC monoliths are well-defined in size. They contribute $0.97 \mathrm{~cm}^{3} \mathrm{~g}^{-1}$ to the total pore volume of the CDC monoliths. In accordance to the nitrogen physisorption experiments, an additional contribution by $1.2 \mathrm{~cm}^{3} \mathrm{~g}^{-1}$ mesopores with a size of $50-4 \mathrm{~nm}$ is detected. Under consideration of the QSDFT cumulative pore volume up to $4 \mathrm{~nm}$ obtained by the nitrogen physisorption experiments (Fig. S3†), the overall pore volume of the CDC monoliths is as high as $3.14 \mathrm{~cm}^{3} \mathrm{~g}^{-1}$. The open meso- and macroporosity of the CDC-Ms is $76.3 \%$ indicating a good accessibility of the microporous walls throughout the entire monoliths.

The macropore architecture of the CDC monoliths can be seen in scanning electron microscopy (SEM) images (Fig. 3). In good accordance to the mercury intrusion porosimetry, the diameter of the wormlike pores is in the range of $0.5-1.0 \mu \mathrm{m}$ and they are surrounded by the micro- and mesoporous CDC walls. The thickness of the latter is in the range of $1 \mu \mathrm{m}$ corresponding to the macropore size of the silica monoliths dictating the pore structure of the replica materials (Fig. S5†).

Due to the large volume of micro- and small mesopores in combination with the highly hydrophobic surface properties typical for $\mathrm{CDCs}^{21}$ and the controllable shape of the adsorbents, CDC-Ms display attractive materials for the removal of hydrophobic organic molecules, such as $n$-butane, from gas mixtures.
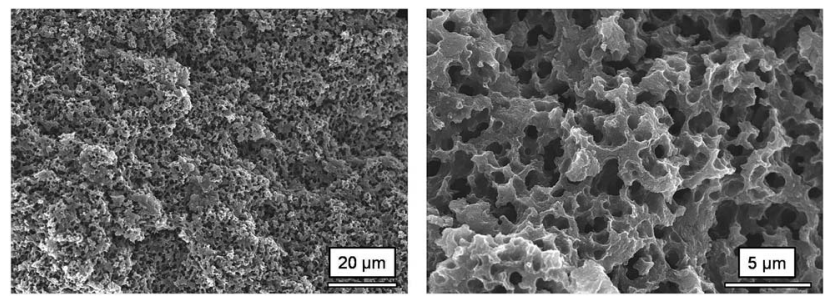

Fig. 3 SEM micrographs of the CDC monoliths.
The presence of the hierarchical pore system might further enhance rapid mass transport throughout the particles which is equally important as the high uptake for any adsorptive filter material.

The volumetric $n$-butane physisorption isotherm of the CDCMs and a purely microporous CDC reference material measured at $5{ }^{\circ} \mathrm{C}$ (Fig. 4, top) are in good accordance with nitrogen physisorption measurements at $-196{ }^{\circ} \mathrm{C}$ (Fig. 1 and S6†). A large uptake and a type IV isotherm including a narrow hysteresis is obtained for the CDC-Ms due to the presence of micro-and narrow mesopores. The microporous material exhibits a type I isotherm with complete pore filling at low relative pressures being also in agreement with the nitrogen physisorption data. The specific surface area and the pore volume of the reference material obtained from nitrogen physisorption are $2342 \mathrm{~m}^{2} \mathrm{~g}^{-1}$ and $1.07 \mathrm{~cm}^{3} \mathrm{~g}^{-1}$, respectively. QSDFT pore size analysis shows a micropore diameter of 0.9-1.0 $\mathrm{nm}$ comparable to the CDC-Ms (Fig. S6†).

Recently, thermal response measurements were identified as an efficient tool for the screening of materials properties such as specific surface area, adsorption capacity, and adsorption kinetics. ${ }^{32}$ In the InfraSORP technology, an IR sensor directly measures the temperature increase caused by the released heat of adsorption in a dynamic flow cell when the inert gas (nitrogen) is switched to the adsorbing gas (typically $n$-butane at $1 \mathrm{bar}$ ). The integrated temperature response signal is proportional to the $n$-butane uptake. Because the relative pressure of
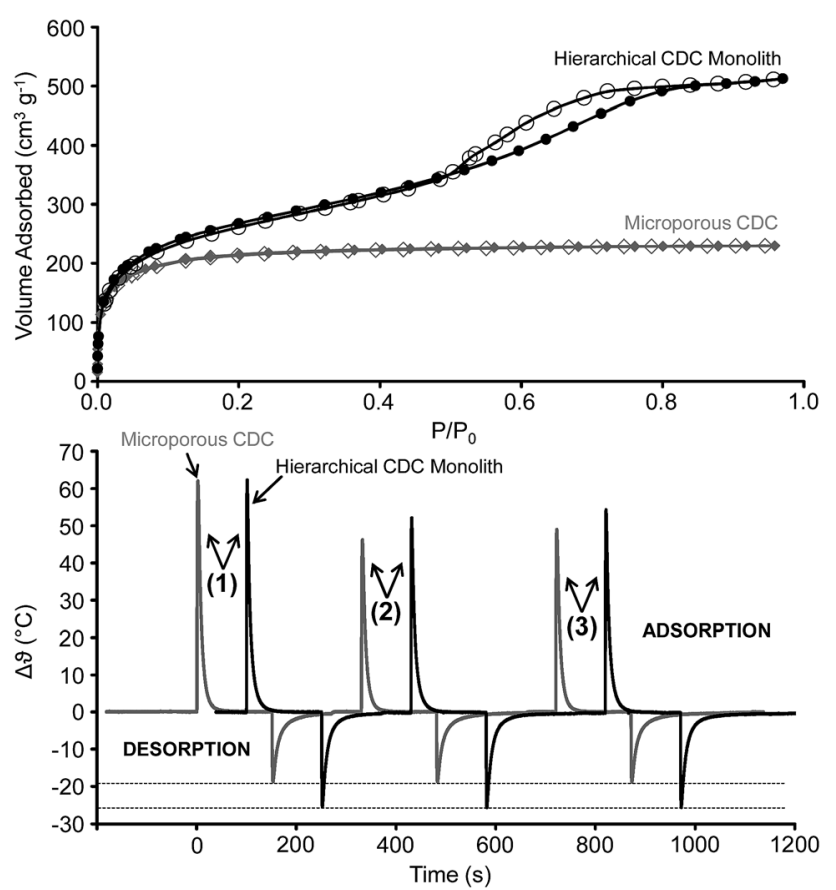

Fig. 4 Top: Volumetric $n$-butane adsorption/desorption (filled symbols/empty symbols) isotherms of the hierarchical CDC-Ms (circles) and a purely microporous $\mathrm{CDC}$ (diamonds) measured at $5^{\circ} \mathrm{C}$ $\left(P_{0} \sim 1.25\right.$ bar). Bottom: thermal response measurements of $n$-butane adsorption $\left(1\right.$ bar, $25^{\circ} \mathrm{C}$ ) in activated (1) hierarchical CDC-Ms (black, horizontally shifted by $100 \mathrm{~s}$ ) and a purely microporous CDC (grey) and after desorption times of $120 \mathrm{~s}(2)$ and $150 \mathrm{~s}$ (3). 
$n$-butane is $P / P_{0} \sim 0.4$ under the applied conditions ( $1 \mathrm{bar}$, $25{ }^{\circ} \mathrm{C}$ ), a significant amount of gas is also adsorbed within the mesopores of the CDC-Ms and therefore the mass-related integrated intensity $\left(\mathrm{A} \mathrm{m}^{-1}\right)$ of the first thermal response peak has a higher value compared to the microporous reference (Fig. S7†). We note that under these conditions, the temperature increase due to the adsorption of the gas in micropores might be higher caused by the larger adsorption potential compared to a mesopore. In consequence, the temperature increase and the mass-related integrated intensity of the signal of the mesoporous silicon carbide material is far below the CDCs due to its lower specific surface area and purely mesoporous structure (Fig. S7†). However, at the same time it gets obvious that there is also a significant contribution to the temperature increase from adsorption in the mesopore system of the CDC-Ms, especially if the higher specific mesopore volume compared to the $\mathrm{SiC}$ monoliths is taken into consideration (Table $\mathrm{S} 1 \dagger$ ).

More important, the measurement demonstrates the higher adsorption/desorption rates in the CDC-Ms as compared to the purely microporous material (Fig. 4, bottom). This is evident from adsorption/desorption cycles starting with fresh activated material and by systematically varying the equilibration time for desorption. After desorption times of 120 and $150 \mathrm{~s}$, the hierarchical monoliths reach as much as $86 \%$ and $88 \%$ of their initial peak area, respectively. In contrast, only $74 \%$ and $79 \%$ of the initial adsorption capacity of the microporous CDC can be utilized after equal desorption time. This indicates slower desorption kinetics for the purely microporous system causing incomplete pore emptying and thus a decrease in signal intensity with decreased equilibration time. Moreover, the rather large decrease in temperature during desorption of $n$-butane from the CDC-Ms indicates a more rapid removal of the molecules in the hierarchical CDC structure. These advanced adsorption kinetics in the CDC-Ms are likely related to (i) the additional mesopores which are not present in the microporous CDCs also contributing the $n$-butane adsorption with advanced accessibility due to their larger diameter and (ii) the better accessibility of the micropores in CDC-Ms through the meso- and macropores present in CDC-Ms. These assumptions are further confirmed by the adsorption/desorption cycling of the purely mesoporous SiC monoliths (Fig. S8†). In contrast to the CDCs, the SiC monoliths reach $100 \%$ of their initial adsorption capacity independently of the elevated desorption time (120 or $150 \mathrm{~s}$ ). Furthermore, the decrease of the temperature during desorption is even larger than the increase during adsorption and equilibrium is reached more rapidly. This indicates extremely rapid desorption of $n$-butane from the SiC monoliths due to their highly accessible mesopores. In summary, the thermal response measurements show the advantage of the pore structure of CDC-Ms in terms of rapid mass transfer due to meso- and macropores combined with high adsorption capacities due to micro- and mesopores. This hierarchical pore structure along with the monolithic shape, well-defined pore sizes, and high purity is highly desirable for efficient adsorption of hydrophobic organic molecules, such as n-butane.

\section{Conclusions}

The synthesis of hierarchical carbide-derived carbon monoliths by direct nanocasting of monolithic silica templates with polycarbosilane precursor was performed. The precise replication of the template pore system in combination with the CDC route leads to the formation of a trimodal micro- meso- and macropore structure including a specific surface area as high as 2662 $\mathrm{m}^{2} \mathrm{~g}^{-1}$ coupled with a total pore volume of $3.14 \mathrm{~cm}^{3} \mathrm{~g}^{-1}$. These CDCs show advanced uptakes and kinetics in the filtration of nbutane rendering CDC-Ms as advanced adsorbents for the removal of volatile organic compounds from gas mixtures. The results demonstrate that structures optimized for mass transport in the liquid phase can be transformed into gas filtration monoliths using the polymer-casting CDC approach due to the integration of additional microporosity under retention of the meso- and macropore system.

\section{Acknowledgements}

We acknowledge financial support within the LOEWE program of excellence of the Federal State of Hessen (project initiative STORE-E).

\section{Notes and references}

1 V. Presser, M. Heon and Y. Gogotsi, Adv. Funct. Mater., 2011, 21, 810.

2 Y. Gogotsi, R. K. Dash, G. Yushin, T. Yildirim, G. Laudisio and J. E. Fischer, J. Am. Chem. Soc., 2005, 127, 16006.

3 M. Oschatz, L. Borchardt, M. Thommes, K. A. Cychosz, I. Senkovska, N. Klein, R. Frind, M. Leistner, V. Presser, Y. Gogotsi and S. Kaskel, Angew. Chem., Int. Ed., 2012, 51, 7577.

4 A. Silvestre-Albero, S. Rico-Francés, F. Rodriguez-Reinoso, A. M. Kern, M. Klumpp, B. J. M. Etzold and J. SilvestreAlbero, Carbon, 2013, 59, 221.

5 L. Borchardt, F. Hasché, M. R. Lohe, M. Oschatz, F. Schmidt, E. Kockrick, C. Ziegler, T. Lescouet, A. Bachmatiuk, B. Büchner, D. Farrusseng, P. Strasser and S. Kaskel, Carbon, 2012, 50, 1861.

6 V. Presser, S.-H. Yeon, C. Vakifahmetoglu, C. A. Howell, S. R. Sandeman, P. Colombo, S. Mikhalovsky and Y. Gogotsi, Adv. Healthcare Mater., 2012, 1, 796.

7 D.-D. Zhou, H.-J. Liu, Y.-G. Wang, C.-X. Wang and Y.-Y. Xia, J. Mater. Chem., 2012, 22, 1937.

8 J. Leis, M. Arulepp, A. Kuura, M. Lätt and E. Lust, Carbon, 2006, 44, 2122.

9 L. Borchardt, M. Oschatz and S. Kaskel, Mater. Horiz., 2014, $1,157$.

10 J. T. Lee, Y. Zhao, S. Thieme, H. Kim, M. Oschatz, L. Borchardt, A. Magasinski, W. I. Cho, S. Kaskel and G. Yushin, Adv. Mater., 2013, 25, 4573.

11 M. Oschatz, L. Borchardt, K. Pinkert, S. Thieme, M. R. Lohe, C. Hoffmann, M. Benusch, F. Wisser, C. Ziegler, L. Giebeler, M. Ruemmeli, J. Eckert, A. Eychmueller and S. Kaskel, Adv. Energy Mater., 2014, 4, 1300645. 
12 A. C. Forse, J. M. Griffin, H. Wang, N. M. Trease, V. Presser, Y. Gogotsi, P. Simon and C. P. Grey, Phys. Chem. Chem. Phys., 2013, 15, 7722.

13 L. Borchardt, M. Oschatz, S. Paasch, S. Kaskel and E. Brunner, Phys. Chem. Chem. Phys., 2013, 15, 15177.

14 M. Oschatz, L. Borchardt, S. Rico-Francés, F. RodríguezReinoso, S. Kaskel and J. Silvestre-Albero, Langmuir, 2013, 29, 8133.

15 J.-S. Bae, T. X. Nguyen and S. K. Bhatia, Carbon, 2014, 68, 531.

16 S. Osswald, J. Chmiola and Y. Gogotsi, Carbon, 2012, 50, 4880.

17 C. R. Perez, S.-H. Yeon, J. Segalini, V. Presser, P.-L. Taberna, P. Simon and Y. Gogotsi, Adv.Funct. Mater., 2013, 23, 1081.

18 J. Chmiola, C. Largeot, P. L. Taberna, P. Simon and Y. Gogotsi, Science, 2010, 328, 480.

19 V. Presser, L. Zhang, J. J. Niu, J. McDonough, C. Perez, H. Fong and Y. Gogotsi, Adv. Energy Mater., 2012, 1, 324.

20 T. Fey, B. Zierath, A. M. Kern, P. Greil and B. J. M. Etzold, Carbon, 2014, 70, 30.

21 M. Oschatz, L. Borchardt, I. Senkovska, N. Klein, M. Leistner and S. Kaskel, Carbon, 2013, 56, 139.
22 E. N. Hoffman, G. Yushin, T. El-Raghy, Y. Gogotsi and M. W. Barsoum, Microporous Mesoporous Mater., 2008, 112, 526.

23 M. Schmirler, T. Knorr, T. Fey, A. Lynen, P. Greil and B. J. M. Etzold, Carbon, 2011, 49, 4359.

24 S. H. Yeon, P. Reddington, Y. Gogotsi, J. E. Fischer, C. Vakifahmetoglu and P. Colombo, Carbon, 2010, 48, 201.

25 L. Chuenchom, R. Kraehnert and B. M. Smarsly, Soft Matter, 2012, 8, 10801.

26 L. Borchardt, M. Oschatz, M. Lohe, V. Presser, Y. Gogotsi and S. Kaskel, Carbon, 2012, 50, 3987.

27 S.-H. Yeon, I. Knoke, Y. Gogotsi and J. E. Fischer, Microporous Mesoporous Mater., 2010, 131, 423.

28 J. Wang, M. Oschatz, T. Biemelt, L. Borchardt, I. Senkovska, M. R. Lohe and S. Kaskel, J. Mater. Chem., 2012, 22, 23893.

29 T. Hara, S. Mascotto, C. Weidmann and B. M. Smarsly, J. Chromatogr. A, 2011, 1218, 3624.

30 P. Adelhelm, K. Cabrera and B. M. Smarsly, Sci. Technol. Adv. Mater., 2012, 13, 1468.

31 Y. S. Hu, P. Adelhelm, B. M. Smarsly, S. Hore, M. Antonietti and M. Maier, Adv. Funct. Mater., 2007, 17, 1873.

32 M. Leistner, W. Grählert and S. Kaskel, Chem. Ing. Tech., 2013, 85, 747. 